\title{
Mutant huntingtin can paradoxically protect neurons from death
}

\author{
T Zuchner $^{\star, 1,2}$ and P Brundin ${ }^{1}$
}

Huntington's disease (HD) is a progressive neurodegenerative disorder caused by a mutation in the gene huntingtin and characterized by motor, cognitive and psychiatric symptoms. Huntingtin contains a CAG repeat in exon 1 . An expansion of this CAG repeat above 35 results in misfolding of Huntingtin, giving rise to protein aggregates and neuronal cell death. There are several transgenic HD mouse models that reproduce most of the features of the human disorder, for example protein inclusions, some neurodegeneration as well as motor and cognitive symptoms. At the same time, a subgroup of the HD transgenic mouse models exhibit dramatically reduced susceptibility to excitotoxicity. The mechanism behind this is unknown. Here, we review the literature regarding this phenomenon, attempt to explain what protein domains are crucial for this phenomenon and point toward a putative mechanism. We suggest, that the C-terminal domain of exon 1 Huntingtin, namely the proline rich domain, is responsible for mediating a neuroprotective effect against excitotoxicity. Furthermore, we point out the possible importance of this mechanism for future therapies in neurological disorders that have been suggested to be associated with excitotoxicity, for example Alzheimer's disease, Parkinson's disease and amyotrophic lateral sclerosis.

Cell Death and Differentiation (2008) 15, 435-442; doi:10.1038/sj.cdd.4402261; published online 2 November 2007

Huntington's disease (HD) is a neurodegenerative disorder that affects approximately 1 in 10000 individuals. Symptoms and signs include depression, personality changes, weight loss, dementia and motor disturbances. The latter are initially characterized by chorea and in the terminal stage they transform into akinesia.

The disease is inherited in an autosomal dominant fashion. The underlying gene mutation was identified in 1993 as a CAG-triplet repeat expansion in the gene huntingtin, which is located on chromosome 4 . $^{1}$ The protein Huntingtin is $3144-$ amino-acid long and is encoded over 67 exons with the CAGtriple repeat located within the first exon. The CAG sequence codes for glutamine and in HD, an expansion of the polyglutamine (poly-Q) stretch, above 35 glutamines results in pathogenicity. The length of the poly- $Q$ stretch inversely correlates with the age of onset of symptoms. ${ }^{2}$ A repeat length of more than 70 glutamines leads to a juvenile form of the disease and the more commonly occurring poly- $Q$ length of $40-55$ typically leads to onset of symptoms at an age of 35-50 years. ${ }^{2}$ Most commonly patients die 15-20 years after the onset of the first symptoms, due to complications of immobilization.
In the end stage of the disease, the total brain weight of $\mathrm{HD}$ patients is reduced by $10-20 \%$. Mainly the caudate nucleus, putamen and neocortex exhibit cell loss and undergo atrophy. Due to the loss of neurons in these brain regions, the lateral ventricles enlarge. The medium-sized spiny neurons are the first to degenerate in the striatum, whereas largeand medium-sized aspiny striatal interneurons are less affected. ${ }^{3,4}$

Although several binding domains (see below for further details) have been identified in Huntingtin, the normal function of the protein is poorly understood. The protein has been suggested to play a role in microtubule-mediated transport and vesicle function. ${ }^{5}$ The pathobiology of mutant Huntingtin is also not well understood. Huntingtin is cleaved and then, in its mutated variant, forms intracellular aggregates, particularly in cell nuclei, and, to a lesser extent, in the cytoplasm, neurites and terminals. ${ }^{6}$ The aggregates are also composed of other proteins, such as transcription factors, chaperones, synaptic proteins and components of the ubiquitin-proteasome pathway. ${ }^{7,8}$ The Huntingtin aggregates have both been suggested to be toxic to the cell as well as neuroprotective. In the latter case, it is argued that the neuroprotective effect of the

\footnotetext{
${ }^{1}$ Neuronal Survival Unit, Department of Experimental Medical Science, Wallenberg Neuroscience Center, Lund University, BMC A10, Lund 22184, Sweden ${ }^{*}$ Corresponding author: T Zuchner, Neuronal Survival Unit, Department of Experimental Medical Science, Wallenberg Neuroscience Center, Lund University, BMC A10, Lund 22184, Sweden.

Tel: + 49341 9731334; Fax: + 49341 9731339; E-mail: Thole.Zuechner@bbz.uni-leipzig.de

${ }^{2}$ Current address: Ultrasensitive Protein Detection Unit, Center for Biotechnology and Biomedicine, Institute for Bioanalytical Chemistry, University of Leipzig, Deutscher Platz 5, Leipzig 04103, Germany

Keywords: Huntington's disease; Huntingtin; excitotoxicity; NMDA; proline rich domain; neuroprotection

Abbreviations: CBS, cystathionine beta-synthase; FIP2, Rab11 family-interacting protein 2; GRB2, growth factor receptor-bound protein 2; HD, Huntington's disease; HIP1, huntingtin interacting protein 1; HIP14, huntingtin interacting protein 14; N-CoR, nuclear receptor corepressor 1; NMDA, N-methyl-D-aspartic acid; PACSIN1, protein kinase $C$ and casein kinase substrate in neurons protein 1; poly-Q, polyglutamine; PSD95, postsynaptic density protein 95; QA, quinolinic acid; RasGAP, RASGTPase-activating protein; $\mathrm{SH} 3$, Src-homology 3

Received 09.5.07; revised 24.9.07; accepted 26.9.07; Edited by M Deshmukh; published online 02.11.07
} 
aggregates is due to conversion of a soluble toxic species into an insoluble form. ${ }^{9}$

\section{Huntingtin Mediates Reduced Susceptibility Against Excitotoxicity}

A large variety of mouse models of HD exist with each expressing different variations of the Huntingtin protein. Several of these have been demonstrated to result in reduced susceptibility against excitotoxicity, and other forms of neural insults, in the mouse brain. Excitotoxicity entails overactivation of glutamate receptors, which leads to neuronal death. ${ }^{10}$ Receptors that can mediate such effects include the $\mathrm{N}$-methyl-D-aspartic acid (NMDA) and alpha-amino-3-hydroxy5-methylisoxazole-4-propionic acid (AMPA) receptors. Quinolinic acid (QA) is an agonist of NMDA receptors and is widely used in experiments to induce excitotoxicity. ${ }^{11}$

In the literature, we have found reports of reduced susceptibility to neural damage in six mouse HD models. We have also identified studies on an additional six models where susceptibility to excitotoxicity was not different from that seen in wild-type control mice. In this review, we describe the models that exhibit neuroprotection, briefly review the changes in the physiology of their brains and, finally, try to explain why some HD mouse models exhibit reduced susceptibility to excitotoxicity while others do not.

In 1999, Hansson et al. ${ }^{12}$ described reduced susceptibility against NMDA receptor-mediated excitotoxicity in the $\mathrm{R} 6 / 1$ mouse model of HD for the first time. The R6/1 mouse model expresses human exon 1 mutant Huntingtin with a poly- $Q$ stretch of around 115 glutamines. Hansson et al. injected $30 \mathrm{nmol}$ QA into the striatum of 18-week-old presymptomatic $\mathrm{R} 6 / 1$ mice and into age-matched controls. The results revealed that cell death in the striatum of $R 6 / 1$ animals was dramatically reduced compared to that seen in wild-type controls (Figure 1). Interestingly, the brains of R6/1 HD mice are not resistant to excitotoxic damage from birth, but the phenomenon develops with age. The temporal development of this resistance was evaluated in another study that examined both $R 6 / 1$ and $R 6 / 2$ mice. ${ }^{13} R 6 / 2$ mice also express human mutant exon 1 Huntingtin, but with an even longer poly- $Q$ stretch of around 150 glutamines. In agreement with findings on earlier symptomatic onset in HD patients with longer poly-Q repeats, ${ }^{14}$ the symptoms appear at a younger age in the $R 6 / 2$ mouse model, with its even longer poly- $Q$ repeat stretch, compared to $\mathrm{R} 6 / 1$ mice. $^{13}$

Interestingly, reduced susceptibility to excitotoxicity develops gradually with age in both $R 6 / 1$ and $R 6 / 2$ mice. The time courses resemble those for the development of motor symptoms in the two models. ${ }^{13}$ For example, in the $R 6 / 1$ mouse, there is no difference in susceptibility against toxin at 3 weeks of age, a partial reduction in susceptibility at 8-13 weeks and complete protection against QA-induced NMDA receptor-mediated excitotoxicity at 22 weeks of age. In R6/2 animals, the protection is complete already at 6 weeks of age. $^{13}$

Eventually it became clear that the R6 mice are not the only transgenic HD mouse models that display reduced susceptibility to excititoxicity. In 2004, Jarabek et al. ${ }^{15}$ reported resistance against QA-induced damage in the $\mathrm{N} 171-82 \mathrm{Q}$ mouse model of HD. This model overexpresses a construct consisting of human exon 1 and parts of exon 2 with an 82-glutamine-long stretch.

In 2005, Slow et al. ${ }^{16}$ examined the 'shortstop' mouse model of HD. This mouse expresses human exons 1 and 2 with a poly- $Q$ stretch of approximately 120 glutamines. At 6 months of age, the striatum was resistant to damage following injections of QA. It is particularly interesting that this mouse model does not exhibit any evidence of neurological dysfunction or neuronal degeneration, although intraneuronal Huntingtin aggregates can be found throughout the brain.

The fourth mouse model found to display reduced susceptibility to excitotoxicity differs from the others in that it overexpresses full-length human Huntingtin with a short non-pathogenic poly-Q stretch (YAC 18). ${ }^{17}$ Thus, this is not really a model of $H D$, in that the poly- $Q$ stretch is of a normal length, but the cells overexpress Huntingtin. Furthermore, the level of protection in the YAC18 model is rather low compared to all other models that show reduced susceptibility: a much lower QA concentration than that used in several other models led only to approximately one-third of the protection observed in all other models. Whatever the mechanism behind the reduced susceptibility against excitotoxicity might be, it is clear that only the mutated form of Huntingtin has the full potential to mediate it.

The same group of investigators then generated additional HD models in which they expressed full-length mutant Huntingtin with 133 glutamine residues and inserted a mutation in either a caspase 3 or a caspase 6 cleavage site. Both the models that lacked a mutation and those with a mutation in the caspase 3 cleavage site were normosensitive to QA, whereas a mutation in the caspase- 6 cleavage site (the model named $\mathrm{C} 6 \mathrm{R}$ ) led to protection against $\mathrm{QA}$-induced excitotoxicity. ${ }^{18}$

Six other transgenic mouse models of HD have been injected with $\mathrm{QA}$ into the striatum and exhibited either
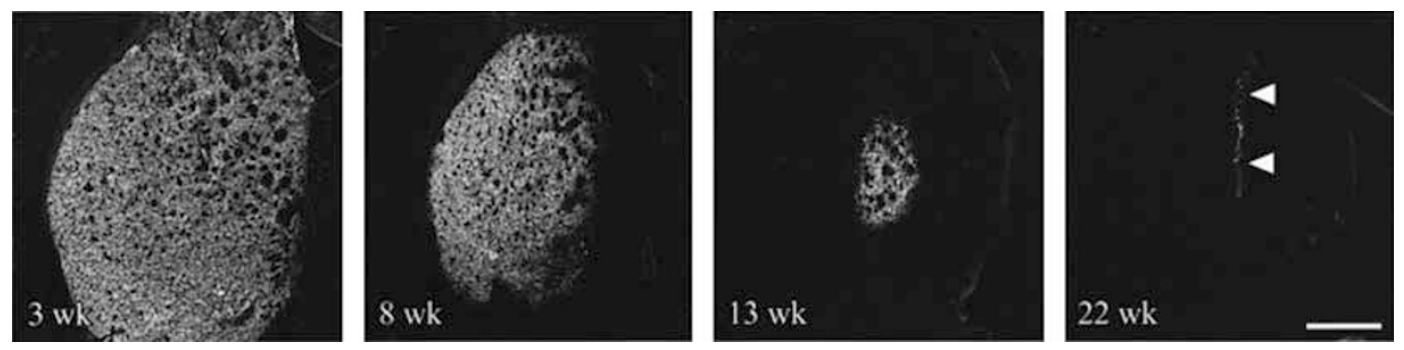

Figure 1 The vulnerability of R6/1 striatal neurons to QA decreases with age. Number of dying neurons was deteced with fluorescent death marker Fluoro-Jade. Arrowheads indicate needle track, scale bar $700 \mu \mathrm{m}$ (from Hansson et al. ${ }^{13}$ ) 
increased or normal sensitivity to the neurotoxin. Two models expressing the full-length huntingtin protein with either 72 or 128 glutamines (YAC72 and YAC128) showed slightly increased sensitivity to $\mathrm{QA}$ compared to matched wild-type control mice. ${ }^{17,19,20}$ In another study, three models using a construct encoding for the first 22 exons of human Huntingtin (equivalent to around $30 \%$ of the protein) with 18,46 or 100 glutamines (tgHD18, tgHD46 and tgHD100) were given intrastriatal injections of QA. ${ }^{21}$ Mice aged up to about 8 months were studied and they all exhibited the same sensitivity to QA as wild-type mice. Finally, injections of malonate into the striatum of a mouse model overexpressing human exons 1-6 Huntingtin with 18 glutamines led to an extent of neuronal death similar to that seen in control mice. ${ }^{22}$

\section{Reduced Susceptibility to Excitotoxins is not Limited to the Striatum}

Several studies have demonstrated that reduced susceptibility to excitotoxins is not limited to the striatum. Moreover, some of the mouse models have been shown to be insensitive to additional toxins. Both $R 6 / 1$ and $R 6 / 2$ mice are protected also against damage induced by intrastriatal injections of malonate, ${ }^{22}$ dopamine and 6-hydroxydopamine. ${ }^{23}$ Furthermore, R6/2 mice are protected against 3-nitroproprionic acid, ${ }^{24}$ kainic acid ${ }^{25}$ and methamphetamine-induced reactive gliosis. ${ }^{26}$ Interestingly, R6/1 mice also exhibit reduced brain damage compared to controls following global cerebral ischemia. $^{27}$ This wide range of reduced susceptibility to neuronal damage makes this phenomenon highly interesting for future research and applications. Moreover, the striatum is not the only brain region that has been shown to be less sensitive to damage in transgenic mouse models of HD. Hansson et al. ${ }^{13}$ showed that the hippocampus of 16-weekold R6/1 mice is also protected against $Q A$ insults. Furthermore, a protection of the hippocampus in R6/1 animals toward global ischemia has been demonstrated. ${ }^{27}$

\section{What are Common Features between Transgenic Mouse HD Models that Display Reduced Sensitivity to Excitotoxins?}

What conclusions can we draw from the studies described above, in an attempt to explain the underlying mechanism responsible for reduced susceptibility to neuronal insults?

One possible explanation for reduced sensitivity to excitotoxins in several HD mouse models could be that the expressed huntingtin fragments, which are expressed in those models throughout development, are highly toxic and may lead to cellular stress. The cellular adaptive response may also provide protection against excitotoxins. However, some observations clearly speak against this hypothesis: first of all, there are also six other transgenic HD models, which are not resistant but express toxic huntingtin constructs. If resistance would occur due to a general stress response, then one would expect also those HD models to display resistance. Furthermore, the shortstop model does not show any neurodegeneration but still shows a high level of resistance. This shows that resistance can also develop without toxic fragments. This argues for a more specific and less general mechanism
Table 1 Transgenic models expressing Huntingtin

\begin{tabular}{|c|c|c|c|c|}
\hline $\begin{array}{l}\text { Mouse } \\
\text { model }\end{array}$ & Exon & $\begin{array}{l}\text { Poly-Q } \\
\text { length }\end{array}$ & [QA] Used & $\begin{array}{c}\% \\
\text { Protection }\end{array}$ \\
\hline $\mathrm{R} 6 / 1$ & 1 & 115 & $30 \mathrm{nmol}$ & $\sim 99$ \\
\hline $\mathrm{R} 6 / 2$ & 1 & 150 & $30 \mathrm{nmol}$ & $\sim 99$ \\
\hline Shortstop & $1+2$ & $\sim 120$ & $6 \mathrm{nmol}$ & $\sim 90$ \\
\hline N171-82Q & $\begin{array}{c}1+\text { part of } \\
2\end{array}$ & 82 & $30 \mathrm{nmol}$ & 78 \\
\hline C6R & $1-67$ & 133 & $4 \mathrm{nmol}$ & $81-91^{*}$ \\
\hline YAC72 & $1-67$ & 72 & $8 \mathrm{nmol}$ & $\begin{array}{c}\text { Not } \\
\text { protected }\end{array}$ \\
\hline YAC128 & $1-67$ & 128 & $6 \mathrm{nmol}$ & $\begin{array}{c}\text { Not } \\
\text { protected }\end{array}$ \\
\hline $\operatorname{tgHD18}$ & $1-22$ & 18 & $30 \mathrm{nmol}$ & $\begin{array}{c}\text { Not } \\
\text { protected }\end{array}$ \\
\hline $\operatorname{tgHD46}$ & $1-22$ & 46 & $30 \mathrm{nmol}$ & $\begin{array}{c}\text { Not } \\
\text { protected }\end{array}$ \\
\hline $\operatorname{tg} \mathrm{HD} 100$ & $1-22$ & 100 & $30 \mathrm{nmol}$ & $\begin{array}{c}\text { Not } \\
\text { protected }\end{array}$ \\
\hline HDex6 & $1-6$ & 18 & $\begin{array}{l}1000 \mathrm{nmol} \\
\text { (Malonate) }\end{array}$ & $\begin{array}{c}\text { Not } \\
\text { protected }\end{array}$ \\
\hline
\end{tabular}

Mouse models resistant to excitotoxicity are shown without background, nonresistant with gray background. Column 'Exon' indicates total length of Huntingtin construct expressed with 67 exons being full-length Huntingtin. *: \% protection depending on comparison to wild type (81\%) or to HD55 (91\%).

behind the observed reduced susceptibility to neuronal insults. In Table 1 and in the following section we summarize factors that may provide insights into underlying mechanisms. When examining the length of the protein that is necessary to induce resistance, it appears that the crucial part of the gene for mediating resistance must be exon 1 . Every mouse model which is resistant expresses at least exon 1, some of them only exon 1 . This suggests that only the protein fragment encoded by exon 1 is necessary to mediate resistance. In the C6R model, which also exhibits resistance, a Caspase- 6 cleavage site located in exon 13 is mutated. ${ }^{18}$ This fact is, at first sight, difficult to reconcile with the concept that exon 1 is crucial and will be discussed later on in this review.

We conclude that one commonality of all resistant mouse models is the expression of exon 1 human huntingtin. At the same time, it is evident that expression of exon 1 huntingtin, even with an expanded poly- $Q$ stretch, does not always lead to resistance. This leads to the conclusion that the precise protein context in which the exon 1 is expressed is vital in deciding over its neuroprotective properties. What other commonalities do the mouse models exhibiting reduced susceptibility have? Looking at the length of the poly- $Q$ stretch, one common property can be found: the length of the poly- $Q$ stretch of strongly protected mouse models is in the pathogenic range. Notably, the length of the poly- $Q$ stretch does influence the development of the reduced susceptibility, as $\mathrm{R} 6 / 2$ mice develop the protective response at much lower ages than $\mathrm{R} 6 / 1$ mice. ${ }^{13}$ The fact that $\mathrm{R} 6$ mice are not resistant to toxin-induced damage from birth also provides a clue to underlying mechanisms. From the R6 models it is clear that the process takes time and that it is not an all-or-nothing phenomenon, but develops gradually. Thus, it appears that either the transgene protein product has to accumulate to a minimal crucial level for it to be effective in this respect or that the protein undergoes conformational changes (e.g. formation of aggregates) once a crucial level is exceeded in the cells. 
Possibly the altered conformation is associated with changes in intracellular location of the huntingtin fragment, another process that can require time to complete. There is a strong correlation between the appearance of intranuclear aggregates of huntingtin at the light microscopical level in the striatum and hippocampus of R6/1 mice and the onset of resistance in different neurons in these regions. ${ }^{13}$ This suggests that this event is either important or that it correlates to another crucial change in the neurons that mediates the reduced susceptibility. In summary, we can conclude that resistance against NMDA receptor-mediated excitotoxicity seems to be related to the part of human Huntingtin encoded by exon 1.

What is the reason that wild-type Huntingtin normally does not mediate resistance? In an attempt to shed more light on this issue we will first shortly review what is already known about the structure of Huntingtin. As we hypothesize exon 1 of the Huntingtin gene is crucial for the neuroprotection phenomenon, we will focus on this structure and its known properties and binding partners.

\section{Structural Aspects and Protein Interactors of Human Exon 1 Huntingtin}

Huntingtin is 3144-amino-acid long and is a multi-domain protein that does not exhibit any significant sequence homology with other known proteins. The first 17 amino acids at the $\mathrm{N}$ terminus have been identified as a nuclear localization signal (NLS). ${ }^{28}$ The poly-Q stretch, which in its mutated form causes $\mathrm{HD}$, is located directly $\mathrm{C}$ terminus of the NLS and is followed by a proline-rich domain. Exon 1 of huntingtin consists of the N-terminal NLS, the poly-Q stretch and an additional 50 amino acids of the protein.

Known interaction partners of exon 1 Huntingtin are GRB2, p53, protein kinase $C$ and casein kinase substrate in neurons protein 1 (PACSIN1), postsynaptic density protein 95 (PSD95), RAS-GTPase-activating protein (RasGAP) and SH3GL3. ${ }^{29-33}$ Other candidates that possibly could interact with the domain of the protein encoded by exon 1, but have not been shown to interact only with this short sequence, include huntingtin interacting protein 1 (HIP1), Rab11 familyinteracting protein 2 (FIP2), huntingtin interacting protein 14 (HIP14), nuclear receptor corepressor 1 (N-CoR) and cystathionine beta-synthase (CBS) ${ }^{34-38}$ (for overview see Figure 2).

What is known about the six proteins that have been found to interact with exon 1 of Huntingtin? First of all, all these proteins interact with the proline-rich domain of Huntingtin, which is localized directly at the $\mathrm{C}$ terminus of the poly- $\mathrm{Q}$ stretch. GRB2 interacts through its Src-homology 3 (SH3) domain with Huntingtin. It activates Ras by forming a complex with EGFR and SOS1. ${ }^{39}$ GRB2 also is involved in the regulation of the $E R K$ pathway. It is not known if the interaction between GRB2 and Huntingtin is dependent on the poly- $Q$ length of Huntingtin. ${ }^{30}$

A GRB2-like protein, SH3GL3 (endophilin 3), also binds to exon 1 Huntingtin. This interaction is discussed as a regulating factor for dynamin and synaptojanin function. ${ }^{29}$ SH3GL3 only interacts with exon 1 Huntingtin if it contains a pathogenic poly- $Q$ stretch. As discussed above, resistance not only occurs in pathogenic Huntingtin, for which reason
SH3GL3 is unlikely to be critically involved in mediating this mechanism.

Another protein that interacts with Huntingtin by a $\mathrm{SH} 3$ domain is PACSIN1. This interaction is poly- $Q$ repeat length dependent and is enhanced in the mutated form with 44 glutamines. ${ }^{32}$ PACSIN1 is believed to play a role in synaptic vesicle recycling.

The tumor suppressor protein p53 has also been found to be one of the interaction partners of exon 1 Huntingtin. ${ }^{31}$ As p53 is critically involved in regulating cell death, it may be involved in mediating reduced susceptibility to excitotoxicity. However, it was also shown ${ }^{31}$ that the length of the poly- $Q$ stretch does not directly influence p53 binding. Indeed, p53 binds to both exon 1 Huntingtin protein constructs either with 20 or 51 glutamines. Interestingly, exon 1 Huntingtin with a 103-long glutamine stretch lacking the proline-rich domain displayed dramatically reduced affinity to $p 53 .^{31}$

RasGAP downregulates the level of Ras-GTP and opposes the effect of GRB2, which was discussed above. ${ }^{40}$ The interaction between RasGAP and Huntingtin might therefore play a role in regulating Ras-GTP levels. Furthermore, it was shown that mice with a null mutation of either the RasGAP or huntingtin gene exhibit increased neuronal apoptosis. ${ }^{41}$

Finally, it is well established that PSD95 (postsynaptic density protein 95 ) interacts with Huntingtin. ${ }^{33}$ PSD95 binds to certain glutamate receptors, that is NMDA- or kainate receptors, and to cytoplasmic signaling proteins such as one member of the above-mentioned RasGAP, SynGAP. As mentioned earlier, the NMDA receptor is a primary mediator of excitotoxicity, especially in response to the NMDA-receptor ligand QA. Huntingtin, through its interaction partners PSD95, RasGAP and GRB2, might be involved in organizing the postsynaptic density and thereby could affect signaling downstream from the synaptic receptors. The N171-82Q transgenic HD mouse, which exhibits reduced sensitivity to excitotoxin, has reduced levels of PSD95. ${ }^{15}$ Sun et al. ${ }^{33}$ reported that a pathogenic poly-Q stretch (48 Q) impairs PSD95 binding to Huntingtin. In the same study, a Huntingtin construct with a non-pathogenic, 16 glutamine long poly- $Q$ stretch protected cells in vitro from glutamate-mediated neuronal death by $82 \%$. In contrast, the same concentration of glutamate led to cell death when cells were transfected with a Huntingtin construct with an expanded (48 Q) poly- $Q$ stretch. Thus, in this case, the mutant huntingtin construct did not mediate neuroprotection, but, on the contrary, it exacerbated excitotoxic death. The Huntingtin constructs used in this particular study spanned the first three exons of the huntingtin gene. This supports the idea that the length of the fragment of huntingtin protein is crucial regarding whether it will be protective or promote cell damage, regardless of the length of the poly- $Q$ stretch. Recall that transgenic mice expressing exon 1 or exons 1 and 2 exhibit reduced susceptibility to excitotoxic damage. In contrast, transgenic mice expressing 22 exons of the huntingtin gene or the full-length protein exhibit normal or increased damage, respectively, following intrastriatal injection of QA. Taken together, PSD95 remains an interesting candidate that could be directly involved in the resistance phenomenon.

Interestingly, all six interacting proteins bind via a $\mathrm{SH} 3$ domain to the proline-rich domain of Huntingtin. For some of 


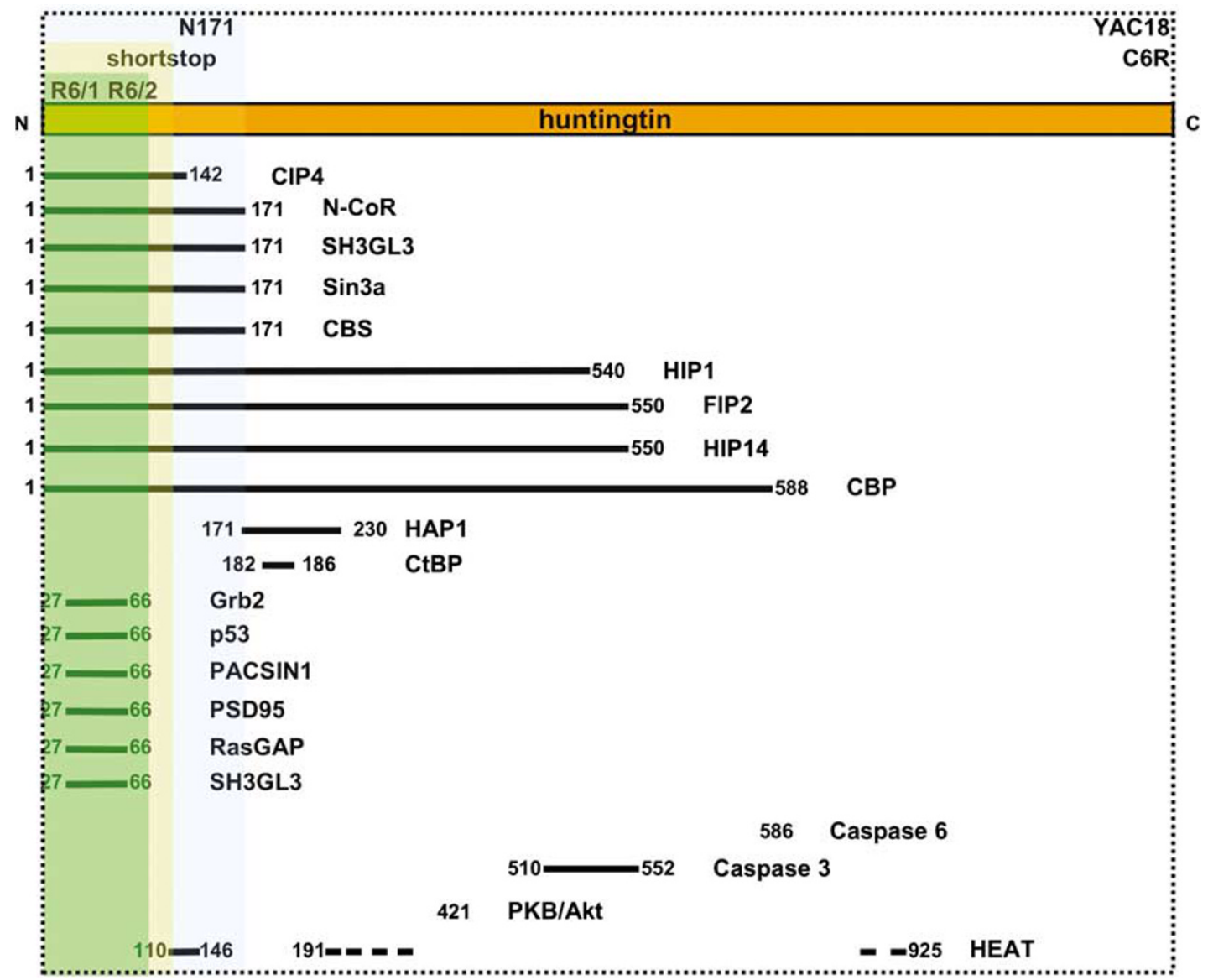

Figure 2 Comparison of Htt domain structure of six HD mouse models that show resistance against excitotoxicity. Different models cover different parts of Htt (full-length $\mathrm{Htt}$ shown in orange, covered parts shown in green, yellow, blue and white) (not in scale). Numbering is based on poly-Q length of 9. CIP4, cdc42-interacting protein 4; N-CoR, nuclear receptor corepressor 1; CBS, cystathionine beta-synthase; HIP1, huntingtin interacting protein 1; FIP2, Rab11 family-interacting protein 2; HIP14, huntingtin interacting protein 14; CBP, CREB binding protein; HAP1, huntingtin-associated protein 1; CtBP, C-terminal-binding protein 1; GRB2, growth factor receptor-bound protein 2; PACSIN1, protein kinase $C$ and casein kinase substrate in neurons protein 1; PSD95, postsynaptic density protein 95; RasGAP, RAS-GTPase-activating protein; PKB/Akt, protein kinase B; HEAT, for Huntingtin, Elongation factor 3, Protein phosphatase 2A, TOR1

them the binding is affected by the length of the poly- $Q$ stretch, whereas for others it is not. PSD95 together with GRB2 and RasGAP are an interesting group of huntingtin interactors, because their functions are related to NMDA receptors. It should be remembered, however, that mouse models expressing Huntingtin fragments do not only display reduced susceptibility to NMDA receptor-mediated excitotoxicity, but are also partially resistant to other insults such as those induced by malonate, ${ }^{22}$ dopamine and 6-hydroxydopamine. ${ }^{23}$ This could imply that the effects of toxic levels of malonate, dopamine and 6-hydroxydopamine are partially mediated via the NMDA receptor, which has been suggested to be the case to varying degrees. ${ }^{22,23}$ Alternatively, the neuroprotective effects elicited by truncated Huntingtin target a cellular pathway that is not specific for NMDA receptor-mediated death.

\section{Possible Mechanisms Involved in Huntingtin-Mediated Resistance}

The reduced neuronal damage seen following injection of toxins into the brains of transgenic HD mice could be classified into a number of fundamentally different mechanisms. First, the neurons might express reduced numbers or impaired variants of glutamate receptors, leading to less calcium entry even when extracellular glutamate levels are high. Second, it is possible that the downstream events that cause cell death following increased levels of cytosolic calcium are defective. Third, the cells may have developed improved defenses against toxin-induced damage, for example higher levels of calcium buffering protein, antioxidant or anti-apoptotic molecules. Fourth, it is even conceivable that the reduced neuronal damage following injections of toxins is related to changes in surrounding glia that more effectively reduce the concentrations of the toxins or produce neuroprotective growth factors. Indeed, glial cells also express the huntingtin transgene in the mouse models that exhibit reduced sensitivity to toxins, so their functions may well be altered. It has been demonstrated in a Drosophila model of HD that selective overexpression of Huntingtin in glia can have a remarkable influence on the whole brain and on disease progression. ${ }^{42}$

Concerning the possible direct involvement of glutamate receptors in the phenomenon, it has been demonstrated that this neuroprotection is not due to a reduced number of 
functional NMDA receptors in R6/1 or R6/2 animals. ${ }^{12}$ In another study, no change in NMDA receptor subunit composition was shown (NR1, NR2A, NR2B). ${ }^{15}$ Nevertheless, the authors were able to observe a decrease in phosphorylation of NR1 at Ser897, previously reported to decrease NMDA receptor current. However, this cannot sufficiently explain the phenomenon observed.

Some earlier studies have examined possible changes in candidate molecules that could explain the neuroprotective effect seen in transgenic mouse models of HD. None of these molecules that have been examined so far are altered in a way that would explain the effect. Thus, there are no major changes in the density and composition of NMDA receptors (as mentioned above) or the levels of brain-derived neurotrophic factor, ciliary neurotrophic factor, ${ }^{43,44}$ Calbindin, ${ }^{12}$ superoxide dismutase, Bcl-XL, X-linked inhibitor of apoptosis, ascorbate and glutathione ${ }^{23}$ in the striatum of R6 mice at ages when they exhibit reduced susceptibility to excitotoxin. Interestingly, calcium levels are reported to recover more rapidly in striatal neurons from 8-week-old R6/2 mice than in wild-type control cells when exposed to $Q A$ in an acute in vitro model system. ${ }^{13}$ Also, basal calcium levels were increased 5- to 6-fold in striatal neurons from R6/2 mice compared to wild-type striatal neurons. The cellular mechanism underlying this difference in calcium handling and its potential importance for the resistance phenomenon remains unclear.

Jarabek et al. ${ }^{15}$ have shown that in N171-82Q HD mice, which display reduced sensitivity to excitotoxin, the levels of PSD95, SAP-102, nNOS, citron and PI-3 kinase are decreased at an age when resistance is developed. These proteins are involved in, for example, synaptic transmission and energy metabolism. The investigators speculated that those decreases could mitigate the detrimental effects of increases in cytosolic calcium. Furthermore, they suggested that observed decreases of dopamine receptor 1 and NR1 phosphorylation could be part of an adaptive response to handle chronically increased calcium levels. They, like others, conclude their findings with the comment that the resistance phenomenon is likely due to a similar mechanism found in ischemic preconditioning. The concept of ischemic preconditioning shows indeed many parallels to the reduced susceptibility in many HD models: after a relatively mild brain ischemia, neurons are less susceptible to severe brain ischemia. ${ }^{45}$ It has been suggested that mild activation of NMDA receptors is a central mechanism of developing ischemic tolerance. ${ }^{46-48}$ Although several other interesting studies have demonstrated potential neuroprotective properties of different factors investigated in the context of HD, none of these factors have afforded the same dramatic protection against excitotoxicity as is in the transgenic HD mouse models we have described in this review article (see e.g. Saydoff et al., ${ }^{49}$ Dedeoglu et al., ${ }^{50}$ McBride et al., ${ }^{51}$ Jin et al. ${ }^{52}$ and Ferrante et al. ${ }^{53}$ ).

\section{Does the Proline-Rich Domain of Huntingtin Cause Resistance?}

As mentioned earlier, there are several interactors for exon 1 huntingtin. It is possible that one or more of them are involved in mediating the resistance of the cells to toxin-induced damage. All of them interact via their SH3 domain with the proline-rich domain of Huntingtin. According to the database available at http://smart.embl-heidelberg.de, there are currently 413 identified proteins with at least one $\mathrm{SH} 3$ domain in Homo sapiens (336 for Mus musculus), most of them are possible interactors with the proline-rich domain of Huntingtin.

One possible explanation why so many different Huntingtin constructs with different properties lead to reduced susceptibility to excitotoxicity is that the poly- $Q$ region does not directly mediate resistance. Instead a domain in the 50-aminoacid-long sequence (still encoded within exon 1 of the gene) located after the poly- $Q$ stretch could be crucial. The poly- $Q$ stretch might influence the secondary and/or tertiary structure of these 50 amino acids so that an amino-acid sequence responsible for mediating resistance is exposed on the surface of the protein. It is also possible that the folding of, for example, full-length huntingtin exposes parts of exon 1 in a different manner than it is in a shorter Huntingtin construct (Figure 3). This could explain why the length of the huntingtin fragment that is expressed can affect whether the resistance phenomenon develops or not. The same concept could explain why the C6R model is resistant whereas the C3R model is not, considering the possibility that a protein that is 512-amino-acid long will fold differently than one that is 585amino-acid long. As a result, different parts of exon 1 could be exposed in the two proteins and thereby lead to an altered interaction with proteins involved in mediating resistance.

There are several experiments that could help determine the mechanism underlying the resistance phenomenon. First of all, the number of possible proline-rich domain interactors is limited, and only a fraction of those are expressed in the brain of HD models that exhibit the resistance to toxins. Moreover, proline-rich domain interactors can readily be identified using, for example, protein-protein chips or yeast two hybrid screens. Viral vectors encoding the identified factors could then be used to examine the effects of overexpressing them in response to toxic insults in vitro. In addition, siRNA or specific small molecule inhibitors could be used to further elucidate the signaling pathways involved in mediating resistance. Finally, it should be possible to create viral vectors that either overexpress the protective protein or otherwise modify the signaling pathway, and could be tested for their neuroprotective effects in animal models of for example Parkinson's disease in vivo. An alternative approach would use the same viral vectors to transduce cells in wild-type animals, examine if they develop the resistance phenomenon, and then identify gene and protein expression changes that could be involved in the mechanism.

In summary, we hypothesize that resistance to NMDA receptor-mediated excitotoxicity is due to factors that bind to the proline-rich domain of huntingtin. Further, we suggest that the accessibility of this region changes due to conformational changes in the parts of the protein encoded by exon 1, and that these are caused by either changes in the poly- $Q$ stretch or in the folding of Huntingtin in general. This could explain the reduced susceptibility found in different HD models with one underlying common mechanism. Clearly, it is still possible that cellular mechanisms underlying resistance in the various HD models differ, but on the whole this seems unlikely. A deeper understanding of the mechanisms mediating reduced 


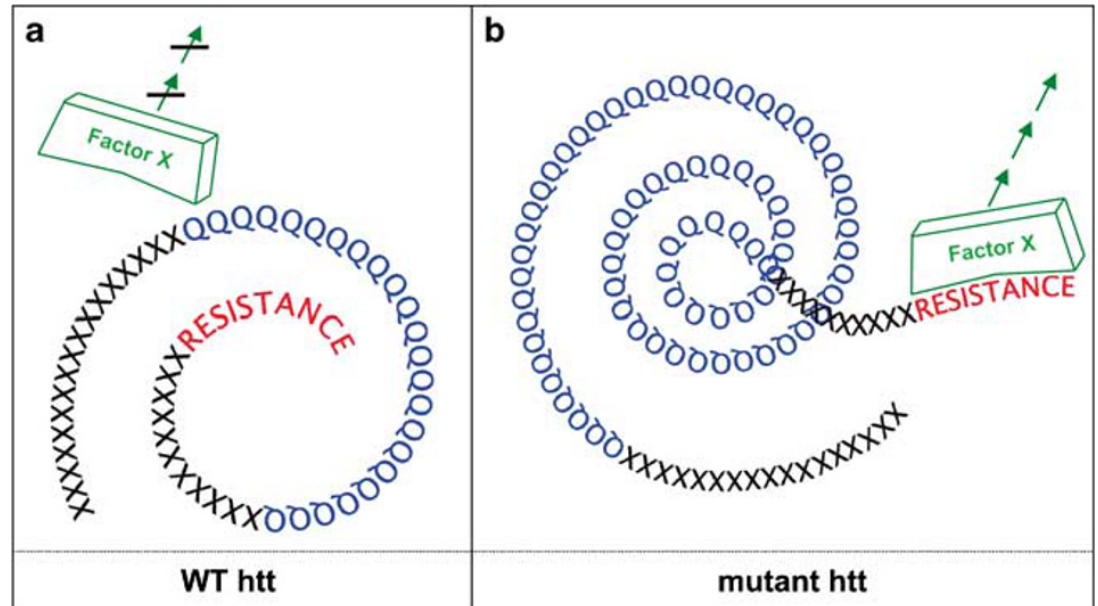

Figure 3 Possible explanation how a prolonged poly- $Q$ stretch could lead to resistance to excitotoxicity. (a) A non-pathogenic poly- $Q$ stretch does not expose a resistance responsible sequence (shown in red) to the protein surface. A factor $X$, responsible for mediating resistance, cannot bind to Huntingtin and cannot signal. (b) The prolonged poly- $Q$ stretch leads to a conformational change of Huntingtin, which exposes the resistance responsible sequence on the protein surface. Factor $X$, responsible for mediating resistance can bind and mediate its effect

susceptibility may mean that we can utilize it in attempts to devise novel neuroprotective strategies against neurodegenerative diseases such as Parkinson's disease, amyotrophic lateral sclerosis, Alzheimer's disease and other poly-Q disorders like SCA1 and SCA3. Of course, it will be essential to separate the beneficial effects of the mutant huntingtin fragment from its possible inherent capacity to cause neurodegeneration. The fact that the Shortstop transgenic mouse model of HD exhibits reduced susceptibility to excitotoxin but no neurological symptoms due to the transgene provides some hope in this regard. Thus, even overexpression of a short huntingtin fragment using a gene therapy approach could prove useful in attempts to stop neurodegenerative disease. It is envisaged that a pharmacological approach could eventually be developed to target the intracellular signaling that is downstream from the primary effect of the mutant huntingtin fragment and thereby trigger a neuroprotective response.

Acknowledgements. We thank Ruben Smith and Mark Denham as well as the anonymous reviewers for helpful comments on the manuscript and Denis Soulet for support with artwork. TZ is supported by The Nordic Centre of Excellence Programme in Molecular Medicine 2004-2009 entitled 'Neurodegeneration'.

1. The Huntington's Disease Collaborative Research Group. A novel gene containing a trinucleotide repeat that is expanded and unstable on Huntington's disease chromosomes. Cell 1993; 72: 971-983.

2. Vonsattel JP, DiFiglia M. Huntington disease. J Neuropathol Exp Neurol 1998; 57 369-384.

3. Vonsattel JP, Stevens TJ, Ferrante RJ, Bird ED, Richardson Jr EP. Neuropathological classification of Huntington's disease. J Neuropath Exp Neurol 1985; 44: 559-577.

4. Ferrante RJ, Beal MF, Richardson Jr EP, Bird ED, Martin JB. Selective sparing of a class of striatal neurons in Huntington's disease. Science 1985; 230: 561-563.

5. Gutekunst CA, Levey Al, Heilman CJ, Whaley WL, Yi H, Nash NR et al. Identification and localization of huntingtin in brain and human lymphoblastoid cell lines with anti-fusion protein antibodies. Proc Natl Acad Sci USA 1995; 92: 8710-8714.

6. Sapp E, Schwarz C, Chase K, Bhide PG, Young AB, Penney J et al. Huntingtin localization in brains of normal and Huntington's disease patients. Ann Neurol 1997; 42: 604-612.

7. Qin ZH, Wang Y, Sapp E, Cuiffo B, Wanker E, Hayden MR et al. Huntingtin bodies sequester vesicle-associated proteins by a polyproline-dependent interaction. $J$ Neurosci 2004; 24: 269-281.
8. Landles C, Bates GP. Huntingtin and the molecular pathogenesis of Huntington's disease. Fourth in molecular medicine review series. EMBO Rep 2004; 5: 958-963.

9. Arrasate M, Mitra S, Schweitzer ES, Segal MR, Finkbeiner S. Inclusion body formation reduces levels of mutant huntingtin and the risk of neuronal death. Nature 2004; 431 : 805-810.

10. Arundine M, Tymianski M. Molecular mechanisms of calcium-dependent neurodegeneration in excitotoxicity. Cell Calcium 2003; 34: 325-337.

11. Schwarcz R, Whetsell Jr WO, Mangano RM. Quinolinic acid: an endogenous metabolite that produces axon-sparing lesions in rat brain. Science 1983; 219: 316-318.

12. Hansson O, Petersen A, Leist M, Nicotera P, Castilho RF, Brundin P. Transgenic mice expressing a Huntington's disease mutation are resistant to quinolinic acid-induced striatal excitotoxicity. Proc Natl Acad Sci USA 1999; 96: 8727-8732.

13. Hansson O, Guatteo E, Mercuri NB, Bernardi G, Li XJ, Castilho RF et al. Resistance to NMDA toxicity correlates with appearance of nuclear inclusions, behavioural deficits and changes in calcium homeostasis in mice transgenic for exon 1 of the huntington gene. Eur $J$ Neurosci 2001; 14: 1492-1504.

14. Snell RG, MacMillan JC, Cheadle JP, Fenton I, Lazarou LP, Davies P et al. Relationship between trinucleotide repeat expansion and phenotypic variation in Huntington's disease. Nat Genet 1993; 4: 393-397.

15. Jarabek BR, Yasuda RP, Wolfe BB. Regulation of proteins affecting NMDA receptorinduced excitotoxicity in a Huntington's mouse model. Brain 2004; 127: 505-516.

16. Slow EJ, Graham RK, Osmand AP, Devon RS, Lu G, Deng Y et al. Absence of behavioral abnormalities and neurodegeneration in vivo despite widespread neuronal huntingtin inclusions. Proc Natl Acad Sci USA 2005; 102: 11402-11407.

17. Leavitt BR, Raamsdonk JM, Shehadeh J, Fernandes H, Murphy Z, Graham RK et al. Wild-type huntingtin protects neurons from excitotoxicity. I Neurochem 2006; 96 : 1121-1129.

18. Graham RK, Deng Y, Slow EJ, Haigh B, Bissada N, Lu G et al. Cleavage at the caspase-6 site is required for neuronal dysfunction and degeneration due to mutant huntingtin. Cell 2006; 125: 1179-1191.

19. Zeron MM, Hansson O, Chen N, Wellington CL, Leavitt BR, Brundin P et al. Increased sensitivity to $\mathrm{N}$-methyl-D-aspartate receptor-mediated excitotoxicity in a mouse model of Huntington's disease. Neuron 2002; 33: 849-860.

20. Shehadeh J, Fernandes HB, Zeron Mullins MM, Graham RK, Leavitt BR, Hayden MR et al. Striatal neuronal apoptosis is preferentially enhanced by NMDA receptor activation in YAC transgenic mouse model of Huntington disease. Neurobiol Dis 2006; 21: 392-403.

21. Petersen A, Mani K, Brundin P. Recent advances on the pathogenesis of Huntington's disease. Exp Neurol 1999; 157: 1-18.

22. Hansson O, Castilho RF, Korhonen L, Lindholm D, Bates GP, Brundin P. Partial resistance to malonate-induced striatal cell death in transgenic mouse models of Huntington's disease is dependent on age and CAG repeat length. J Neurochem 2001; 78: 694-703.

23. Petersen A, Hansson O, Puschban Z, Sapp E, Romero N, Castilho RF et al. Mice transgenic for exon 1 of the Huntington's disease gene display reduced striatal sensitivity to neurotoxicity induced by dopamine and 6-hydroxydopamine. Eur J Neurosci 2001; 14: $1425-1435$

24. Hickey MA, Morton AJ. Mice transgenic for the Huntington's disease mutation are resistant to chronic 3-nitropropionic acid-induced striatal toxicity. J Neurochem 2000; 75 . 2163-2171. 
25. Morton AJ, Leavens W. Mice transgenic for the human Huntington's disease mutation have reduced sensitivity to kainic acid toxicity. Brain Res Bull 2000; 52: 51-59.

26. MacGibbon GA, Hamilton LC, Crocker SF, Costain WJ, Murphy KM, Robertson HA et al Immediate-early gene response to methamphetamine, haloperidol, and quinolinic acid is not impaired in Huntington's disease transgenic mice. J Neurosci Res 2002; 67: 372-378.

27. Schiefer J, Alberty A, Dose T, Oliva S, Noth J, Kosinski CM. Huntington's disease transgenic mice are resistant to global cerebral ischemia. Neurosci Lett 2002; 334: 99-102.

28. Cornett J, Cao F, Wang CE, Ross CA, Bates GP, Li SH et al. Polyglutamine expansion of huntingtin impairs its nuclear export. Nat Genet 2005; 37: 198-204.

29. Sittler A, Walter S, Wedemeyer N, Hasenbank R, Scherzinger E, Eickhoff $\mathrm{H}$ et al. SH3GL3 associates with the Huntingtin exon 1 protein and promotes the formation of polyglncontaining protein aggregates. Mol Cell 1998; 2: 427-436.

30. Liu YF, Deth RC, Devys D. SH3 domain-dependent association of huntingtin with epidermal growth factor receptor signaling complexes. J Biol Chem 1997; 272: 8121-8124.

31. Steffan JS, Kazantsev A, Spasic-Boskovic O, Greenwald M, Zhu YZ, Gohler H et al. The Huntington's disease protein interacts with p53 and CREB-binding protein and represses transcription. Proc Natl Acad Sci USA 2000; 97: 6763-6768.

32. Modregger J, DiProspero NA, Charles V, Tagle DA, Plomann M. PACSIN 1 interacts with huntingtin and is absent from synaptic varicosities in presymptomatic Huntington's disease brains. Hum Mol Genet 2002; 11: 2547-2558.

33. Sun Y, Savanenin A, Reddy PH, Liu YF. Polyglutamine-expanded huntingtin promotes sensitization of $\mathrm{N}$-methyl-D-aspartate receptors via post-synaptic density 95 . J Biol Chem 2001; 276: 24713-24718.

34. Boutell JM, Wood JD, Harper PS, Jones AL. Huntingtin interacts with cystathionine betasynthase. Hum Mol Genet 1998; 7: 371-378.

35. Singaraja RR, Hadano S, Metzler M, Givan S, Wellington CL, Warby S et al. HIP14, a nove ankyrin domain-containing protein, links huntingtin to intracellular trafficking and endocytosis. Hum Mol Genet 2002; 11: 2815-2828.

36. Wanker EE, Rovira C, Scherzinger E, Hasenbank R, Walter S, Tait D et al. HIP-I: a huntingtin interacting protein isolated by the yeast two-hybrid system. Hum Mol Genet 1997; 6: 487-495.

37. Hattula K, Peranen J. FIP-2, a coiled-coil protein, links Huntingtin to Rab8 and modulates cellular morphogenesis. Curr Biol 2000; 10: 1603-1606.

38. Boutell JM, Thomas P, Neal JW, Weston VJ, Duce J, Harper PS et al. Aberrant interactions of transcriptional repressor proteins with the Huntington's disease gene product, huntingtin. Hum Mol Genet 1999; 8: 1647-1655.

39. Lowenstein EJ, Daly RJ, Batzer AG, Li W, Margolis B, Lammers R et al. The SH2 and SH3 domain-containing protein GRB2 links receptor tyrosine kinases to ras signaling. Cell 1992 70: $431-442$.
40. Ellis CMM, McCormick F, Pawson T. Phosphorylation of GAP and GAP-associated proteins by transforming and mitogenic tyrosine kinases. Nature 1990; 343: 377-381.

41. Henkemeyer MRD, Holmyard DP, Puri MC, Mbamula G, Harpal K, Shih TS et al. Vascular system defects and neuronal apoptosis in mice lacking ras GTPase-activating protein. Nature 1995; 377: 695-701.

42. Lievens JC, Rival T, Iche M, Chneiweiss H, Birman S. Expanded polyglutamine peptides disrupt EGF receptor signaling and glutamate transporter expression in Drosophila. Hum Mol Genet 2005; 14: 713-724

43. Petersen AA, Larsen KE, Behr GG, Romero N, Przedborski S, Brundin P et al. Brainderived neurotrophic factor inhibits apoptosis and dopamine-induced free radical production in striatal neurons but does not prevent cell death. Brain Res Bull 2001; 56 : 331-335.

44. Petersen A, Brundin P. Effects of ciliary neurotrophic factor on excitotoxicity and calciumionophore A23187-induced cell death in cultured embryonic striatal neurons. Exp Neurol 1999; 160: 402-412.

45. Kitagawa K, Matsumoto M, Tagaya M, Hata R, Ueda H, Niinobe $M$ et al. 'Ischemic tolerance' phenomenon found in the brain. Brain Res 1990; 528: 21-24.

46. Bond A, Lodge D, Hicks CA, Ward MA, O'Neill MJ. NMDA receptor antagonism, but not AMPA receptor antagonism attenuates induced ischaemic tolerance in the gerbil hippocampus. Eur J Pharmacol 1999; 380: 91-99.

47. Grabb MC, Choi DW. Ischemic tolerance in murine cortical cell culture: critical role for NMDA receptors. J Neurosci 1999; 19: 1657-1662.

48. Kato H, Liu Y, Araki T, Kogure K. MK-801, but not anisomycin, inhibits the induction of tolerance to ischemia in the gerbil hippocampus. Neurosci Lett 1992; 139: 118-121.

49. Saydoff JA, Garcia RA, Browne SE, Liu L, Sheng J, Brenneman D et al. Oral uridine prodrug PN401 is neuroprotective in the R6/2 and N171-82Q mouse models of Huntington's disease. Neurobiol Dis 2006; 24: 455-465.

50. Dedeoglu A, Ferrante RJ, Andreassen OA, Dillmann WH, Beal MF. Mice overexpressing 70-kDa heat shock protein show increased resistance to malonate and 3-nitropropionic acid. Exp Neurol 2002; 176: 262-265.

51. McBride JL, Ramaswamy S, Gasmi M, Bartus RT, Herzog CD, Brandon EP et al. Viral delivery of glial cell line-derived neurotrophic factor improves behavior and protects striatal neurons in a mouse model of Huntington's disease. Proc Natl Acad Sci USA 2006; 103 : 9345-9350.

52. Jin K, LaFevre-Bernt M, Sun Y, Chen S, Gafni J, Crippen D et al. FGF-2 promotes neurogenesis and neuroprotection and prolongs survival in a transgenic mouse model of Huntington's disease. Proc Natl Acad Sci USA 2005; 102: 18189-18194.

53. Ferrante RJ, Ryu H, Kubilus JK, D’Mello S, Sugars KL, Lee J et al. Chemotherapy for the brain: the antitumor antibiotic mithramycin prolongs survival in a mouse model of Huntington's disease. J Neurosci 2004; 24: 10335-10342. 